\title{
Algorithms for Quantum Branching Programs Based on Fingerprinting
}

\author{
Farid Ablayev* \\ Institute for Informatics \\ Kazan, Russian Federation \\ fablayev@gmail.com
}

\author{
Alexander Vasiliev ${ }^{\dagger}$ \\ Institute for Informatics \\ Kazan, Russian Federation \\ Alexander.Vasiliev@ksu.ru
}

\begin{abstract}
In the paper we develop a method for constructing quantum algorithms for computing Boolean functions by quantum ordered read-once branching programs (quantum OBDDs). Our method is based on fingerprinting technique and representation of Boolean functions by their characteristic polynomials. We use circuit notation for branching programs for desired algorithms presentation. For several known functions our approach provides optimal QOBDDs. Namely we consider such functions as $M O D_{m}, E Q_{n}$, Palindrome $_{n}$, and $P E R M_{n}$ (testing whether given Boolean matrix is the Permutation Matrix). We also propose a generalization of our method and apply it to the Boolean variant of the Hidden Subgroup Problem.
\end{abstract}

\section{Introduction}

During the last two decades different types of quantum computation models based on Turing Machines, automata, and circuits have been considered. For some of them different examples of functions were presented for which quantum models appear to be much more (exponentially) efficient than their classical counterparts.

In this paper we consider a restricted model of computation known as Ordered Read-Once Quantum Branching Programs. In computer science this model is also known as Ordered Binary Decision Diagrams (OBDDs). The main reason for the investigation of restricted models of quantum computers was proposed by Ambainis and Freivalds in 1998 [1]. Considering one-way quantum finite automata, they suggested that first quantum-mechanical computers would consist of a comparatively simple and fast quantum-mechanical part connected to a classical computer.

Two models of quantum branching programs were introduced by Ablayev, Gainutdinova, Karpinski [2] (leveled programs), and by Nakanishi, Hamaguchi, Kashiwabara [11] (non-leveled programs). Later it was shown by Sauerhoff [12] that these two models are polynomially equivalent.

For this model we develop the fingerprinting technique introduced in [6]. The basic ideas of this approach are due to Freivalds (e.g. see the book [10]). It was later successfully applied in the quantum automata setting by Ambainis and Freivalds in 1998 [1] (later improved in [5]). Subsequently, the same technique was adapted for the quantum branching programs by Ablayev, Gainutdinova and Karpinski in 2001 [2], and was later generalized in [6].

For our technique we use the presentation of Boolean functions known as characteristic polynomials. Our definition of the characteristic polynomial differs from that of [4], though it uses similar ideas.

We display several known functions for which our method provides optimal QOBDDs. Namely, these functions are $M O D_{m}, E Q_{n}$, Palindrome ${ }_{n}$, and $P E R M_{n}$.

\footnotetext{
${ }^{*}$ Work was in part supported by the Russian Foundation for Basic Research under the grant 08-07-00449a
}

${ }^{\dagger}$ Work was in part supported by the Russian Foundation for Basic Research under the grant 08-07-00449a

S. Barry Cooper \& Vincent Danos (eds.): Fifth Workshop on Developments in Computational Models-Computational Models From Nature EPTCS 9, 2009, pp. 1-11, doi 10.4204/EPTCS.9.1 (c) F. Ablayev \& A. Vasiliev

This work is licensed under the Creative Commons Attribution License. 


\section{Preliminaries}

We use the notation $|i\rangle$ for the vector from $\mathcal{H}^{d}$, which has a 1 on the $i$-th position and 0 elsewhere. Obviously, the set of vectors $|1\rangle, \ldots,|d\rangle$ forms an orthonormal basis in $\mathcal{H}^{d}$.

Definition 1. A Quantum Branching Program $Q$ over the Hilbert space $\mathcal{H}^{d}$ is defined as

$$
\left.Q=\left\langle T, \mid \psi_{0}\right\rangle, M_{\text {accept }}\right\rangle,
$$

where $T$ is a sequence of $l$ instructions: $T_{j}=\left(x_{i_{j}}, U_{j}(0), U_{j}(1)\right)$ is determined by the variable $x_{i_{j}}$ tested on the step $j$, and $U_{j}(0), U_{j}(1)$ are unitary transformations in $\mathcal{H}^{d}$.

Vectors $|\psi\rangle \in \mathcal{H}^{d}$ are called states (state vectors) of $Q,\left|\psi_{0}\right\rangle \in \mathcal{H}^{d}$ is the initial state of $Q$, and $M_{\text {accept }}$ - is a projector on the accepting subspace $\mathcal{H}_{\text {accept }}^{d}$ (i.e. it is a diagonal zero-one projection matrix, which determines the final projective measurement).

We define a computation of $Q$ on an input $\sigma=\left(\sigma_{1}, \ldots, \sigma_{n}\right) \in\{0,1\}^{n}$ as follows:

1. A computation of $Q$ starts from the initial state $\left|\psi_{0}\right\rangle$;

2. The $j$-th instruction of $Q$ reads the input symbol $\sigma_{i_{j}}$ (the value of $x_{i_{j}}$ ) and applies the transition matrix $U_{j}=U_{j}\left(\sigma_{i_{j}}\right)$ to the current state $|\psi\rangle$ to obtain the state $\left|\psi^{\prime}\right\rangle=U_{j}\left(\sigma_{i_{j}}\right)|\psi\rangle$;

3. The final state is

$$
\left|\psi_{\sigma}\right\rangle=\left(\prod_{j=l}^{1} U_{j}\left(\sigma_{i_{j}}\right)\right)\left|\psi_{0}\right\rangle .
$$

4. After the l-th (last) step of quantum transformation $Q$ measures its configuration $\left|\psi_{\sigma}\right\rangle$, and the input $\sigma$ is accepted with probability

$$
\operatorname{Pr}_{\text {accept }}(\sigma)=\left\langle\psi_{\sigma} M_{\text {accept }}^{\dagger} \mid M_{\text {accept }} \psi_{\sigma}\right\rangle=\| M_{\text {accept }}\left|\psi_{\sigma}\right\rangle \|_{2}^{2}
$$

Circuit representation. A QBP can be viewed as a quantum circuit aided with an ability to read classical bits as control variables for unitary operations. That is any quantum circuit is a QBP which does not depend essentially on its classical inputs.

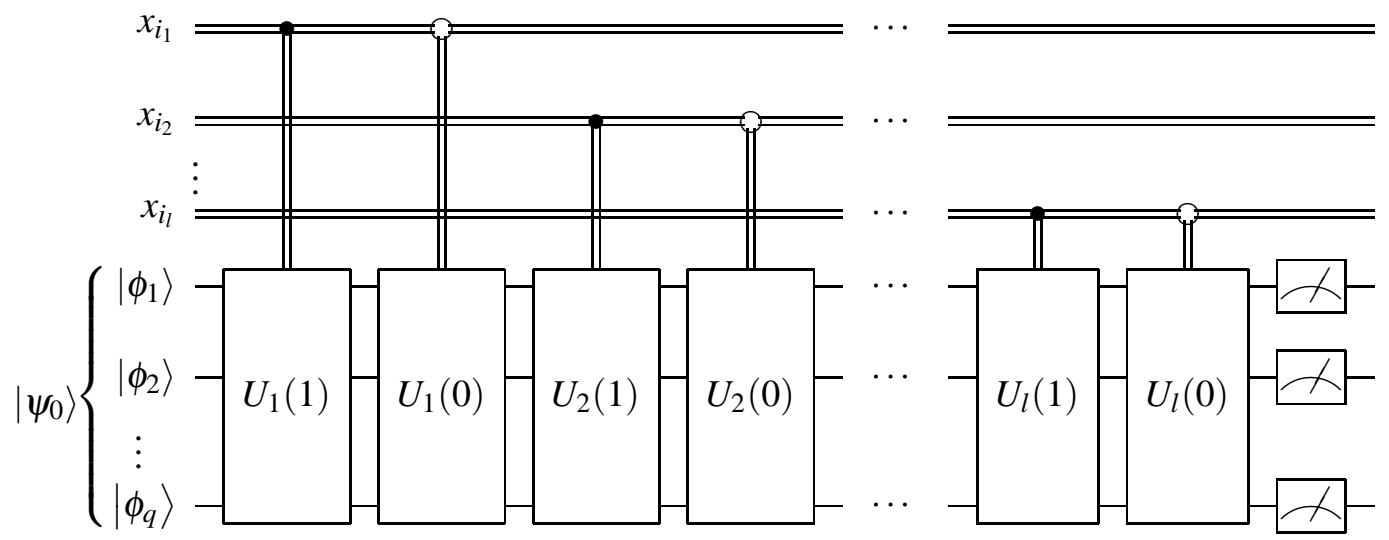

Here $x_{i_{1}}, \ldots, x_{i_{l}}$ is the sequence of (not necessarily distinct) variables denoting classical control bits. Using the common notation single wires carry quantum information and double wires denote classical information and control. 
Complexity measures. The width of $Q$ is the dimension $d$ of the state space $\mathcal{H}^{d}$, the length of $Q$ is the number $l$ of instructions in the sequence $T$.

Note that for a QBP in the circuit setting another important complexity measure explicitly comes out - a number $q$ of qubits physically needed to implement a corresponding quantum system with classical control. From definition it follows that $\log d \leq q$.

Definition 2. We call a quantum branching program a q-qubit $Q B P$, if it can be implemented as a classically-controlled quantum system based on q qubits.

Acceptance criteria. A QBP $Q$ computes the Boolean function $f$ with one-sided error if there exists an $\varepsilon \in(0,1)$ (called an error) such that for all $\sigma \in f^{-1}(1)$ the probability of $Q$ accepting $\sigma$ is 1 and for all $\sigma \in f^{-1}(0)$ the probability of $Q$ erroneously accepting $\sigma$ is less than $\varepsilon$.

\section{Read-once branching programs.}

Definition 3. We call a $\mathrm{QBP} Q$ a quantum $\mathrm{OBDD}$ (QOBDD) or read-once $\mathrm{QBP}$ if each variable $x \in$ $\left\{x_{1}, \ldots, x_{n}\right\}$ occurs in the sequence $T$ of transformations of $Q$ at most once.

For the rest of the paper we're only interested in QOBDDs, i.e. the length of all programs would be $n$ (the number of input variables).

Generalized Lower Bound. The following general lower bound on the width of QOBDDs was proven in [3].

Theorem 1. Let $f\left(x_{1}, \ldots, x_{n}\right)$ be a Boolean function computed by a quantum read-once branching program $Q$. Then

$$
\operatorname{width}(Q)=\Omega(\log \text { width }(P))
$$

where $P$ is a deterministic OBDD of minimal width computing $f\left(x_{1}, \ldots, x_{n}\right)$.

That is, the width of a quantum OBDD cannot be asymptotically less than logarithm of the width of the minimal deterministic OBDD computing the same function. And since the deterministic width of many "natural" functions is exponential [13], we obtain the linear lower bound for these functions.

\section{Algorithms for QBPs Based on Fingerprinting}

Generally [10], fingerprinting - is a technique that allows to present objects (words over some finite alphabet) by their fingerprints, which are significantly smaller than the originals. It is used in randomized and quantum algorithms to test equality of some objects (binary strings) with one-sided error by simply comparing their fingerprints.

In this paper we develop a variant of the fingerprinting technique adapted for quantum branching programs. At the heart of the method is the representation of Boolean functions by polynomials of special type, which we call characteristic.

\subsection{Characteristic Polynomials for Quantum Fingerprinting}

We call a polynomial $g\left(x_{1}, \ldots, x_{n}\right)$ over the ring $\mathbb{Z}_{m}$ a characteristic polynomial of a Boolean function $f\left(x_{1}, \ldots, x_{n}\right)$ and denote it $g_{f}$ when for all $\sigma \in\{0,1\}^{n} g_{f}(\sigma)=0$ iff $f(\sigma)=1$.

Lemma 1. For any Boolean function $f$ there exists a characteristic polynomial $g_{f}$ over $\mathbb{Z}_{2^{n}}$. 
Proof. One way to construct such characteristic polynomial $g_{f}$ is transforming a sum of products representation for $\neg f$.

Let $K_{1} \vee \ldots \vee K_{l}$ be a sum of products for $\neg f$ and let $\tilde{K}_{i}$ be a product of terms from $K_{i}$ (negations $\neg x_{j}$ are replaced by $1-x_{j}$ ). Then $\tilde{K}_{1}+\ldots+\tilde{K}_{l}$ is a characteristic polynomial over $\mathbb{Z}_{2^{n}}$ for $f$ since it equals 0 $\Longleftrightarrow$ all of $\tilde{K}_{i}$ (and thus $K_{i}$ ) equal 0 . This happens only when the negation of $f$ equals 0 .

Generally, there are many polynomials for the same function. For example, the function $E Q_{n}$, which tests the equality of two $n$-bit binary strings, has the following polynomial over $\mathbb{Z}_{2^{n}}$ :

$$
\sum_{i=1}^{n}\left(x_{i}\left(1-y_{i}\right)+\left(1-x_{i}\right) y_{i}\right)=\sum_{i=1}^{n}\left(x_{i}+y_{i}-2 x_{i} y_{i}\right) .
$$

On the other hand, the same function can be represented by the polynomial

$$
\sum_{i=1}^{n} x_{i} 2^{i-1}-\sum_{i=1}^{n} y_{i} 2^{i-1}
$$

We use this presentation of Boolean functions for our fingerprinting technique which generalizes the algorithm for $M O D_{m}$ function by Ambainis and Nahimovs [5].

\subsection{Fingerprinting technique}

For a Boolean function $f$ we choose an error rate $\varepsilon>0$ and pick a characteristic polynomial $g$ over the ring $\mathbb{Z}_{m}$. Then for arbitrary binary string $\sigma=\sigma_{1} \ldots \sigma_{n}$ we create its fingerprint $\left|h_{\sigma}\right\rangle$ composing $t=2^{\lceil\log ((2 / \varepsilon) \ln 2 m)\rceil}$ single qubit fingerprints $\left|h_{\sigma}^{i}\right\rangle$ :

$$
\begin{aligned}
\left|h_{\sigma}^{i}\right\rangle & =\cos \frac{2 \pi k_{i} g(\sigma)}{m}|0\rangle+\sin \frac{2 \pi k_{i} g(\sigma)}{m}|1\rangle \\
\left|h_{\sigma}\right\rangle & =\frac{1}{\sqrt{t}} \sum_{i=1}^{t}|i\rangle\left|h_{\sigma}^{i}\right\rangle
\end{aligned}
$$

That is, the last qubit is rotated by $t$ different angles about the $\hat{y}$ axis of the Bloch sphere.

The chosen parameters $k_{i} \in\{1, \ldots, m-1\}$ for $i \in\{1, \ldots, t\}$ are "good" following the notion of [1].

Definition 4. A set of parameters $K=\left\{k_{1}, \ldots, k_{t}\right\}$ is called "good" for some integer $b \neq 0$ mod $m$ if

$$
\frac{1}{t^{2}}\left(\sum_{i=1}^{t} \cos \frac{2 \pi k_{i} b}{m}\right)^{2}<\varepsilon .
$$

The left side of inequality is the squared amplitude of the basis state $|0\rangle^{\otimes \log t}|0\rangle$ if $b=g(\sigma)$ and the operator $H^{\otimes \log t} \otimes I$ has been applied to the fingerprint $\left|h_{\sigma}\right\rangle$. Informally, that kind of set guarantees, that the probability of error will be bounded by a constant below 1 .

The following lemma proves the existence of a "good" set and generalizes the proof of the corresponding statement from [5].

Lemma 2. [6] There is a set $K$ with $|K|=t=2^{\lceil\log ((2 / \varepsilon) \ln 2 m)\rceil}$ which is "good" for all integer $b \neq$ $0 \bmod m$.

We use this result for our fingerprinting technique choosing the set $K=\left\{k_{1}, \ldots, k_{t}\right\}$ which is "good" for all $b=g(\sigma) \neq 0$. That is, it allows to distinguish those inputs whose image is 0 modulo $m$ from the others. 


\subsection{Boolean Functions Computable via Fingerprinting Method}

Let $f\left(x_{1}, \ldots, x_{n}\right)$ be a Boolean function and $g$ be its characteristic polynomial. The following theorem holds.

Theorem 2. Let $\varepsilon \in(0,1)$. If $g$ is a linear polynomial over $\mathbb{Z}_{m}$, i.e. $g=c_{1} x_{1}+\ldots c_{n} x_{n}+c_{0}$, then $f$ can be computed with one-sided error $\varepsilon$ by a quantum $O B D D$ of width $O\left(\frac{\log m}{\varepsilon}\right)$.

Proof. Here is the algorithm in the circuit notation:

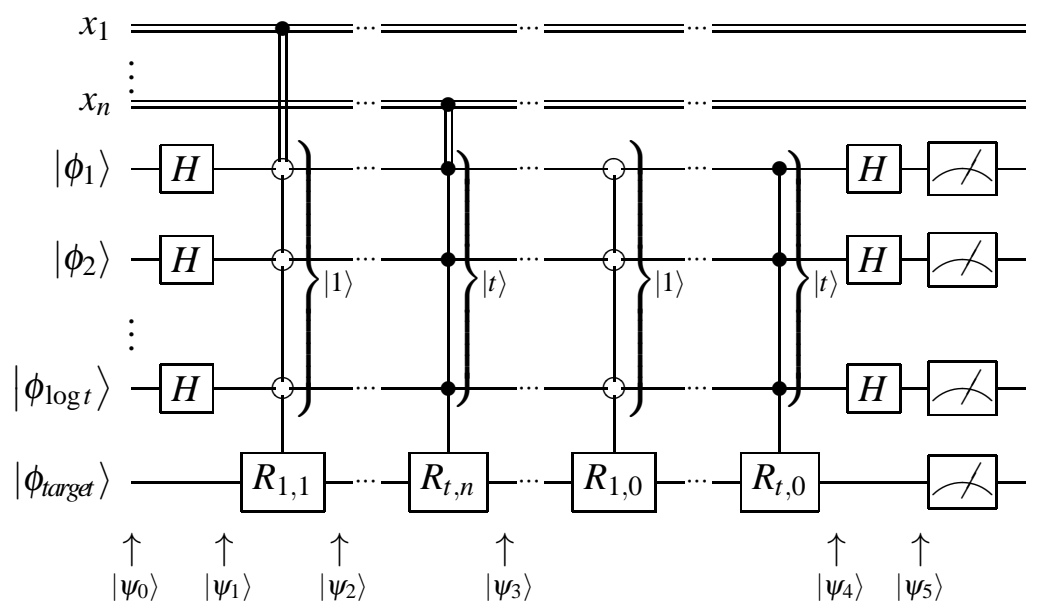

Initially qubits $\left|\phi_{1}\right\rangle \otimes\left|\phi_{2}\right\rangle \otimes \cdots \otimes\left|\phi_{\log t}\right\rangle \otimes\left|\phi_{\text {target }}\right\rangle$ are in the state $\left|\psi_{0}\right\rangle=|0\rangle^{\otimes \log t}|0\rangle$. For $i \in\{1, \ldots, t\}$, $j \in\{0, \ldots, n\}$ we define rotations $R_{i, j}$ as

$$
R_{i, j}=R_{\hat{y}}\left(\frac{4 \pi k_{i} c_{j}}{m}\right)
$$

where $c_{j}$ are the coefficients of the linear polynomial for $f$ and the set of parameters $K=\left\{k_{1}, \ldots, k_{t}\right\}$ is "good" according to the Definition 4 with $t=2^{\lceil\log ((2 / \varepsilon) \ln 2 \cdot m)\rceil}$.

Let $\sigma=\sigma_{1} \ldots \sigma_{n} \in\{0,1\}^{n}$ be an input string.

The first layer of Hadamard operators transforms the state $\left|\psi_{0}\right\rangle$ into

$$
\left|\psi_{1}\right\rangle=\frac{1}{\sqrt{t}} \sum_{i=1}^{t}|i\rangle|0\rangle
$$

Next, upon input symbol 0 identity transformation $I$ is applied. But if the value of $x_{j}$ is 1 , then the state of the last qubit is transformed by the operator $R_{i, j}$, rotating it by the angle proportional to $c_{j}$. Moreover, the rotation is done in each of $t$ subspaces with the corresponding amplitude $1 / \sqrt{t}$. Such a parallelism is implemented by the controlled operators $C_{i}\left(R_{i, j}\right)$, which transform the states $|i\rangle|\cdot\rangle$ into $|i\rangle R_{i, j}|\cdot\rangle$, and leave others unchanged. For instance, having read the input symbol $x_{1}=1$, the system would evolve into state

$$
\begin{aligned}
\left|\psi_{2}\right\rangle & =\frac{1}{\sqrt{t}} \sum_{i=1}^{t} C_{i}\left(R_{i, 1}\right)|i\rangle|0\rangle=\frac{1}{\sqrt{t}} \sum_{i=1}^{t}|i\rangle R_{i, 1}|0\rangle \\
& =\frac{1}{\sqrt{t}} \sum_{i=1}^{t}|i\rangle\left(\cos \frac{2 \pi k_{i} c_{1}}{m}|0\rangle+\sin \frac{2 \pi k_{i} c_{1}}{m}|1\rangle\right)
\end{aligned}
$$


Thus, after having read the input $\sigma$ the amplitudes would "collect" the sum $\sum_{j=1}^{n} c_{j} \sigma_{j}$

$$
\left|\psi_{3}\right\rangle=\frac{1}{\sqrt{t}} \sum_{i=1}^{t}|i\rangle\left(\cos \frac{2 \pi k_{i} \sum_{j=1}^{n} c_{j} \sigma_{j}}{m}|0\rangle+\sin \frac{2 \pi k_{i} \sum_{j=1}^{n} c_{j} \sigma_{j}}{m}|1\rangle\right)
$$

At the next step we perform the rotations by the angle $\frac{4 \pi k_{i} c_{0}}{m}$ about the $\hat{y}$ axis of the Bloch sphere for each $i \in\{1, \ldots, t\}$. Therefore, the state of the system would be

$$
\left|\psi_{4}\right\rangle=\frac{1}{\sqrt{t}} \sum_{i=1}^{t}|i\rangle\left(\cos \frac{2 \pi k_{i} g(\sigma)}{m}|0\rangle+\sin \frac{2 \pi k_{i} g(\sigma)}{m}|1\rangle\right) .
$$

Applying $H^{\otimes \log t} \otimes I$ we obtain the state

$$
\begin{aligned}
\left|\psi_{5}\right\rangle= & \left(\frac{1}{t} \sum_{i=1}^{t} \cos \frac{2 \pi k_{i} g(\sigma)}{m}\right)|0\rangle^{\otimes \log t}|0\rangle+ \\
& +\gamma|0\rangle^{\otimes \log t}|1\rangle+\sum_{i=2}^{t}|i\rangle\left(\alpha_{i}|0\rangle+\beta_{i}|1\rangle\right),
\end{aligned}
$$

where $\gamma, \alpha_{i}$, and $\beta_{i}$ are some unimportant amplitudes.

The input $\sigma$ is accepted if the measurement outcome is $|0\rangle^{\otimes \log t}|0\rangle$. Clearly, the accepting probability is

$$
\operatorname{Pr}_{\text {accept }}(\sigma)=\frac{1}{t^{2}}\left(\sum_{i=1}^{t} \cos \frac{2 \pi k_{i} g(\sigma)}{2^{n}}\right)^{2} .
$$

If $f(\sigma)=1$ then $g(\sigma)=0$ and the program accepts $\sigma$ with probability 1 . Otherwise, the choice of the set $K=\left\{k_{1}, \ldots, k_{t}\right\}$ guarantees that

$$
\operatorname{Pr}_{\text {accept }}(\sigma)=\frac{1}{t^{2}}\left(\sum_{i=1}^{t} \cos \frac{2 \pi k_{i} g(\sigma)}{2^{n}}\right)^{2}<\varepsilon
$$

Thus, $f$ can be computed by a $q$-qubit quantum OBDD, where $q=\log 2 t=O(\log \log m)$. The width of the program is $2^{q}=O(\log m)$.

The following functions have the aforementioned linear polynomials and thus are effectively computed via the fingerprinting technique.

$M O D_{m}$ The function $M O D_{m}$ tests whether the number of 1's in the input is 0 modulo $m$. The linear polynomial over $\mathbb{Z}_{m}$ for this function is

$$
\sum_{i=1}^{n} x_{i}
$$

The lower bound for the width of deterministic OBDDs computing this function is $\Omega(m)$ [13]. Thus, our method provides an exponential advantage of quantum OBDD over any deterministic one.

$E Q_{n}$ The function $E Q_{n}$, which tests the equality of two $n$-bit binary strings, has the following polynomial over $\mathbb{Z}_{2^{n}}$

$$
\sum_{i=1}^{n} x_{i} 2^{i-1}-\sum_{i=1}^{n} y_{i} 2^{i-1}
$$


Palindrome $_{n}\left(x_{1}, \ldots, x_{n}\right)$ This function tests the symmetry of the input, i.e. whether $x_{1} x_{2} \ldots x_{\lfloor n / 2\rfloor}=$ $x_{n} x_{n-1} \ldots x_{\lceil n / 2\rceil+1}$ or not. The polynomial over $\mathbb{Z}_{2^{\lfloor n / 2\rfloor}}$ is

$$
\sum_{i=1}^{\lfloor n / 2\rfloor} x_{i} 2^{i-1}-\sum_{i=\lceil n / 2\rceil}^{n} x_{i} 2^{n-i}
$$

$P E R M_{n}$ The Permutation Matrix test function $\left(P E R M_{n}\right)$ is defined on $n^{2}$ variables $x_{i j}(1 \leq i, j \leq n)$. It tests whether the input matrix contains exactly one 1 in each row and each column. Here is a polynomial over $\mathbb{Z}_{(n+1)^{2 n}}$

$$
\sum_{i=1}^{n} \sum_{j=1}^{n} x_{i j}\left((n+1)^{i-1}+(n+1)^{n+j-1}\right)-\sum_{i=1}^{2 n}(n+1)^{i-1}
$$

Note, that this function cannot be effectively computed by a deterministic OBDD - the lower bound is $\Omega\left(2^{n} n^{-5 / 2}\right)$ regardless of the variable ordering [13]. The width of the best known probabilistic OBDD, computing this function with one-sided error, is $O\left(n^{4} \log n\right)$ [13]. Our algorithm has the width $O(n \log n)$. Since the lower bound $\Omega(n-\log n)$ follows from Theorem 1 , our algorithm is almost optimal.

The following table provides the comparison of the width of quantum and deterministic OBDDs for the aforementioned functions.

\begin{tabular}{|l|l|l|}
\hline & OBDD & QOBDD \\
\hline$M O D_{m}$ & $\Omega(m)$ & $O(\log m)$ \\
\hline$E Q_{n}$ & $2^{\Omega(n)}$ & $O(n)$ \\
\hline Palindrome $_{n}$ & $2^{\Omega(n)}$ & $O(n)$ \\
\hline PERM $_{n}$ & $\Omega\left(2^{n} n^{-5 / 2}\right)$ & $O(n \log n)$ \\
\hline
\end{tabular}

\section{Generalized Approach}

The fingerprinting technique described in the previous section allows us to test a single property of the input encoded by a characteristic polynomial. Using the same ideas we can test the conjunction of several conditions encoded by a group of characteristic polynomials which we call a characteristic of a function.

Definition 5. We call a set $\chi_{f}^{m}$ of polynomials over $\mathbb{Z}_{m}$ a characteristic of a Boolean function $f$ if for all polynomials $g \in \chi_{f}^{m}$ and all $\sigma \in\{0,1\}^{n}$ it holds that $g(\sigma)=0$ iff $\sigma \in f^{-1}(1)$.

We say that a characteristic is linear if all of its polynomials are linear.

From Lemma 1 it follows that for each Boolean function there is always a characteristic consisting of a single characteristic polynomial.

Now we can generalize the Fingerprinting technique from section 3.2 .

Generalized Fingerprinting technique For a Boolean function $f$ we choose an error rate $\varepsilon>0$ and pick a characteristic $\chi_{f}^{m}=\left\{g_{1}, \ldots, g_{l}\right\}$. Then for arbitrary binary string $\sigma=\sigma_{1} \ldots \sigma_{n}$ we create its fingerprint $\left|h_{\sigma}\right\rangle$ composing $t \cdot l\left(t=2^{\lceil\log ((2 / \varepsilon) \ln 2 m)\rceil}\right)$ single qubit fingerprints $\left|h_{\sigma}^{i}(j)\right\rangle$ :

$$
\begin{aligned}
\left|h_{\sigma}^{i}(j)\right\rangle & =\cos \frac{\pi k_{i} g_{j}(\sigma)}{m}|0\rangle+\sin \frac{\pi k_{i} g_{j}(\sigma)}{m}|1\rangle \\
\left|h_{\sigma}\right\rangle & =\frac{1}{\sqrt{t}} \sum_{i=1}^{t}|i\rangle\left|h_{\sigma}^{i}(1)\right\rangle\left|h_{\sigma}^{i}(2)\right\rangle \ldots\left|h_{\sigma}^{i}(l)\right\rangle
\end{aligned}
$$


Theorem 3. If $\chi_{f}^{m}$ is a linear characteristic then $f$ can be computed by a quantum OBDD of width $O\left(2^{\left|\chi_{f}^{m}\right|} \log m\right)$.

Proof. Here is the sketch of the algorithm:

1. Upon the input $\sigma=\sigma_{1} \ldots \sigma_{n}$ we create the fingerprint $\left|h_{\sigma}\right\rangle$.

2. We measure $\left|h_{\sigma}\right\rangle$ in the standard computational basis and accept the input if the outcome of the last $l$ qubits is the all-zero state. Thus, the probability of accepting $\sigma$ is

$$
\operatorname{Pr}_{\text {accept }}(\sigma)=\frac{1}{t} \sum_{i=1}^{t} \cos ^{2} \frac{\pi k_{i} g_{1}(\sigma)}{m} \cdots \cos ^{2} \frac{\pi k_{i} g_{l}(\sigma)}{m}
$$

If $f(\sigma)=1$ then all of $g_{i}(\sigma)=0$ and we will always accept.

If $f(\sigma)=0$ then there is at least one such $j$ that $g_{j}(\sigma) \neq 0$ and the choice of the "good" set $K$ guarantees that the probability of the erroneously accepting is bounded by

$$
\begin{aligned}
\operatorname{Pr}_{\text {accept }}(\sigma) & =\frac{1}{t} \sum_{i=1}^{t} \cos ^{2} \frac{\pi k_{i} g_{1}(\sigma)}{m} \cdots \cos ^{2} \frac{\pi k_{i} g_{l}(\sigma)}{m} \\
& \leq \frac{1}{t} \sum_{i=1}^{t} \cos ^{2} \frac{\pi k_{i} g_{j}(\sigma)}{m}=\frac{1}{t} \sum_{i=1}^{t} \frac{1}{2}\left(1+\cos \frac{2 \pi k_{i} g_{j}(\sigma)}{m}\right) \\
& =\frac{1}{2}+\frac{1}{2 t} \sum_{i=1}^{t} \cos \frac{2 \pi k_{i} g_{j}(\sigma)}{m} \\
& \leq \frac{1}{2}+\frac{\sqrt{\varepsilon}}{2}
\end{aligned}
$$

The number of qubits used by this QBP is $q=O(\log \log m+l), l=\left|\chi_{f}^{m}\right|$. Therefore, the width of the program is $2^{q}=O\left(2^{\left|\chi_{f}^{m}\right|} \log m\right)$.

The generalized approach can be used to construct an effective quantum OBDD for the Boolean variant of the Hidden Subgroup Problem.

\subsection{The upper bound for Hidden Subgroup Function}

This problem was first defined and considered in [8], where the following Boolean variant of the Hidden Subgroup Problem was defined.

Definition 6. Let $K$ be a normal subgroup of a finite group $G$. Let $X$ be a finite set. For a sequence $\chi \in X^{|G|}$ let $\sigma=\operatorname{bin}(\chi)$ be its representation in binary. If $\sigma$ encodes no correct sequence $\chi=\chi_{1} \ldots \chi_{|G|}$, then Hidden Subgroup function of $\sigma$ is set to be zero, otherwise:

$$
H S P_{G, K}(\sigma)= \begin{cases}1, & \text { if } \forall a \in G \forall i, j \in a K\left(\chi_{i}=\chi_{j}\right) \\ & \text { and } \forall a, b \in G \forall i \in a K \forall j \in b K\left(a K \neq b K \Rightarrow \chi_{i} \neq \chi_{j}\right) ; \\ 0, & \text { otherwise. }\end{cases}
$$

Let $f$ be the function encoded by the input sequence. We want to know if a function $f: G \rightarrow X$ "hides" the subgroup $K$ in the group $G$. Our program receives $G$ and $K$ as parameters, and function $f$ as an input string containing values of $f$ it takes on $G$. The values are arranged in lexicographical order. See Definition 6 . 
We make two assumptions. First, we assume that the set $X$ contains exactly $(G: K)$ elements. Indeed, having read the function $f$, encoded in the input sequence $\sigma$, we have $X$ to be the set of all different values that $f$ takes. Obviously, if $|X|$ is less or greater than $(G: K)$, then $\operatorname{HSP}_{G, K}(\sigma)=0$. The second assumption, is that we replace all values of $f$ by numbers from 1 through $(G: K)$. Thus, $\operatorname{HSP}_{G, K}\left(x_{1}, \ldots, x_{n}\right)$ is a Boolean function of $n=|G|\lceil\log G: K\rceil$ variables. In these two assumptions the following theorem holds.

Theorem 4. Function $H S P_{G, K}(x)$ can be computed with one-sided error by a quantum OBDD of width $O(n)$.

Proof. First we shall prove the following lemma.

Lemma 3. In order to correctly compute $\operatorname{HSP}_{G, K}(x)$ it is enough to perform following calculations.

1. For every coset we check equalities for all input sequence values that have indices from this coset;

2. From every coset we choose a representative, and check if the sum of values of $f$ on all the representatives equals to the following value

$$
S=\sum_{i=1}^{G: K} i=\frac{(G: K)((G: K)+1)}{2} .
$$

Proof. One direction is straightforward. The other direction is also not difficult. Suppose we have the two conditions of the lemma satisfied. Let $a K$ and $b K$ be two different cosets with elements $d \in a K$ and $c \in b K$, such that $\sigma_{d}=\sigma_{c}$. We fix $c \in b K$. There are two cases possible:

1. For all $d \in a K\left(\sigma_{d}=\sigma_{c}\right)$;

2. There exists $d^{\prime} \in a K\left(\sigma_{d} \neq \sigma_{c}\right)$.

Apparently in the first case we indeed could choose any of the elements of a coset to check inequalities. In the second case the first condition of the lemma would fail. The reasoning for $b K$ is analogous.

When the values of $f$ are different on different cosets, obviously, the sum of these values is the sum of numbers from 1 through $G: K$. Therefore, $\operatorname{HSP}_{G, K}(\sigma)=1$ iff both conditions of the lemma are satisfied.

According to the previous lemma, $\operatorname{HSP}_{G, K}(x)$ has a characteristic consisting of two polynomials over $\mathbb{Z}_{2^{n}}$, checking conditions of the lemma. We shall construct them explicitly to show they are linear.

We shall adopt another indexation of $\chi$ when convenient: $\chi_{a, q}$ is a value of $f$ on the $q$-th element of the coset $a K$.

Therefore, for a binary input symbol $x_{j}$ we define

- $a=a(j)$ for the number of the corresponding coset;

- $q=q(j)$ for the number of the corresponding element of the coset $a$;

- $r=r(j)$ for the number of bit in the binary representation of $\chi_{a, q}$

and start indexation from 0 . Thus $a \in\{0, \ldots,(G: K)-1\}, q \in\{0, \ldots,|a K|-1\}$.

In this notation the polynomials are:

1. $g_{1}(x)=\sum_{a} \sum_{q} 2^{(|K| a+q)\lceil\log G: K\rceil}\left(\chi_{a, q}-\chi_{a, q-1 \bmod |K|}\right)$. Thus, $g_{1}(x)=0$ iff for every coset $a$ function $f$ maps all the elements of $a$ onto the same element of $X$.

2. $g_{2}(x)=\left(\sum_{j=1}^{(G: K)} \chi_{i_{j}}\right)-S$, where $\chi_{i_{j}}$ is the representative chosen from the $j$-th coset. Therefore, $g_{2}(x)$ checks whether the images of elements from different cosets are distinct.

By the generalized fingerprinting technique we can construct quantum OBDD of width $O(n)$, computing $\operatorname{HSP}_{G, K}(x)$ with one-sided error. 


\section{References}

[1] A. Ambainis and R. Freivalds (1998): 1-way quantum finite automata: strengths, weaknesses and generalization. Proceeding of the 39th IEEE Conference on Foundation of Computer Science, pp. 332-342. See also arXiv:quant-ph/9802062 v3.

[2] F. Ablayev, A. Gainutdinova and M. Karpinski (2001): On computational power of quantum branching programs. Lecture Notes in Computer Science 2138, pp. 59-70. Springer-Verlag. See also arXiv:quant$\mathrm{ph} / 0302022 \mathrm{v} 1$.

[3] F. Ablayev, A. Gainutdinova, M. Karpinski, C. Moore, and C. Pollette (2005): On the computational power of probabilistic and quantum branching programs of constant width. Information and Computation.

[4] V.D. Agrawal, D. Lee and H. Wozniakowski (1998): Numerical Computation of Characteristic Polynomials of Boolean Functions and its Applications. Numerical Algorithms 17, pp. 261-278.

[5] A. Ambainis and N. Nahimovs (2008): Improved constructions of quantum automata. arXiv:0805.1686v1 Available at http://xxx.lanl.gov/archive/quant-ph.

[6] F. Ablayev and A. Vasiliev (2008): On the Computation of Boolean Functions by Quantum Branching Programs via Fingerprinting. Electronic Colloquium on Computational Complexity, TR08-059. Available at http://www.eccc.uni-trier.de/eccc/.

[7] R. Freivalds (1979): Fast probabilistic algorithms. FCT'79, Lecture Notes in Computer Science 74, pp. 57-69. Springer-Verlag.

[8] A. Khasianov (2005): Complexity Bounds On Some Fundamental Computational Problems For Quantum Branching Programs. Ph.D. thesis, Bonn University. Available at http://nbn-resolving.de/urn:nbn: de: $\mathrm{hbz}: 5 \mathrm{~N}-05696$.

[9] C. Moore and J.P. Crutchfield (2000): Quantum automata and quantum grammars. Theoretical Computer Science 237, pp. 275-306.

[10] R. Motwani and P. Raghavan (1995): Randomized Algorithms. Cambridge University Press.

[11] M. Nakanishi, K. Hamaguchi and T. Kashiwabara (2000): Ordered quantum branching programs are more powerful than ordered probabilistic branching programs under a bounded-width restriction. Proceedings of the 6th Annual International Conference on Computing and Combinatorics, Lecture Notes in Computer Science 1858, pp. 467-476.

[12] M. Sauerhoff and D. Sieling (2004): Quantum branching programs and space-bounded nonuniform quantum complexity. Theoretical Computer Science 334, pp. 177-225.

[13] I. Wegener (2000): Branching programs and binary decision diagrams. SIAM Monographs on Discrete Mathematics and Applications. SIAM Press. 


\section{A Proof of Lemma 2}

Proof. Using Azuma's inequality (see, e.g., [10]) we prove that a random choice of the set $K$ is "good" with positive probability . $1\}$.

Let $1 \leq g \leq m-1$ and let $K$ be the set of $t$ parameters selected uniformly at random from $\{0, \ldots, m-$

We define random variables $X_{i}=\cos \frac{2 \pi k_{i} g}{m}$ and $Y_{k}=\sum_{i=1}^{k} X_{i}$. We want to prove that Azuma's inequality is applicable to the sequence $Y_{0}=0, Y_{1}, Y_{2}, Y_{3}, \ldots$, i.e. it is a martingale with bounded differences. First, we need to prove that $E\left[Y_{k}\right]<\infty$.

From the definition of $X_{i}$ it follows that

$$
E\left[X_{i}\right]=\frac{1}{m} \sum_{j=0}^{m-1} \cos \frac{2 \pi j g}{m}
$$

Consider the following weighted sum of $m$ th roots of unity

$$
\frac{1}{m} \sum_{j=0}^{m-1} \exp \left(\frac{2 \pi j g}{m} i\right)=\frac{1}{m} \cdot \frac{\exp (2 \pi i g m / m)-1}{\exp (2 \pi i g / m)-1}=0,
$$

since $g$ is not a multiple of $m$.

$E\left[X_{i}\right]$ is exactly the real part of the previous sum and thus is equal to 0 .

Consequently, $E\left[Y_{k}\right]=\sum_{i=1}^{k} E\left[X_{i}\right]=0<\infty$.

Second, we need to show that the conditional expected value of the next observation, given all the past observations, is equal to the last observation.

$$
E\left[Y_{k+1} \mid Y_{1}, \ldots, Y_{k}\right]=\frac{1}{m} \sum_{j=0}^{m-1}\left(Y_{k}+\cos \frac{2 \pi j g}{m}\right)=Y_{k}+\frac{1}{m} \sum_{j=0}^{m-1} \cos \frac{2 \pi j g}{m}=Y_{k}
$$

Since $\left|Y_{k+1}-Y_{k}\right|=\left|X_{k+1}\right| \leq 1$ for $k \geq 0$ we apply Azuma's inequality to obtain

$$
\operatorname{Pr}\left(\left|Y_{t}-Y_{0}\right| \geq \lambda\right)=\operatorname{Pr}\left(\left|\sum_{i=1}^{t} X_{i}\right| \geq \lambda\right) \leq 2 \exp \left(-\frac{\lambda^{2}}{2 t}\right)
$$

Therefore, we induce that the probability of $K$ being not "good" for $1 \leq g \leq m-1$ is at most

$$
\operatorname{Pr}\left(\left|\sum_{i=1}^{t} X_{i}\right| \geq \sqrt{\varepsilon} t\right) \leq 2 \exp \left(-\frac{\varepsilon t}{2}\right) \leq \frac{1}{m}
$$

for $t=\lceil(2 / \varepsilon) \ln 2 m\rceil$.

Hence the probability that constructed set is not "good" for at least one $1 \leq g \leq m-1$ is at most $(m-1) / m<1$. Therefore, there exists a set which is "good" for all $1 \leq g \leq m-1$. This set will also be "good" for all $g \neq 0 \bmod m$ because $\cos \frac{2 \pi k(g+j m)}{m}=\cos \frac{2 \pi k g}{m}$. 